\title{
Knee Anatomy and Biomechanics and its Relevance to Knee Replacement
}

\author{
Vera Pinskerova and Pavel Vavrik
}

\subsection{What Is the Normal Knee Biomechanics?}

From the late 1960s to the early 1990s, when much of the original design work on knee replacement prostheses was carried out, the kinematics of the knee were universally understood to involve a rigid 4-bar link mechanism. It was understood that as the knee flexed, this mechanism caused both femoral condyles to roll back across the top of the tibia and then to roll forward with extension. Because this was thought to be a normal feature of knee flexion/extension, tibial components were made relatively unconstrained anteroposteriorly, thus permitting "roll back/ forward." The concept of the 4-bar link mechanism originated with the work of Zuppinger [1]. The concept became part of received orthopedic knowledge, perhaps as a consequence of its appearance in a number of widely used textbooks.

In 1941 Brantigan and Voshell [2] reported that "the medial femoral condyle acts as the axis of rotation of the knee joint." They based this conclusion on their observation that the medial meniscus hardly moves anteroposteriorly in flexion, whereas the lateral meniscus moves backward. From the late 1990s onward, a number of investigators have used MRI and other tech-

\footnotetext{
V. Pinskerova $(\varangle) \cdot$ P. Vavrik

1st Orthopaedic Clinic, 1st Faculty of Medicine, Charles University, Prague, Czech Republic

e-mail: vera.pinskerova@lf1.cuni.cz
}

niques to demonstrate that Brantigan and Voshell were correct: the medial femoral condyle hardly moves anteroposteriorly between $0^{\circ}$ and $120^{\circ}$, whereas the lateral femoral condyle moves about $20 \mathrm{~mm}$ anteroposteriorly over that arc. The arc from $120^{\circ}$ to full flexion follows a different kinematic regime.

The shapes of the articulating surfaces: The sagittal and coronal shapes and modes of articulation observed using MRI have been confirmed by dissection and cryosection [3], by 3D digitization [4], and by CT [5]. The following description of the shape of the articulating bones is based on that work. The surface shapes in sagittal section are relevant to flexion/extension: when circular femoral surfaces contact the tibia, pure flexion may be thought to occur around their centers. Medial femorotibial compartment: The articular surface of the medial femoral condyle in sagittal section can be regarded as posteriorly circular (the flexion facet, FF, center: the FFC, see Fig. 14.1a) with an average radius of about $22 \mathrm{~mm}$ subtending an arc of $110^{\circ}$. The extreme posterior portion of the condyle (about $24^{\circ}$ of arc) is of a smaller radius, but this portion contacts only the posterior horn (in extreme flexion), never the tibia itself, and is therefore not part of the direct tibiofemoral articulation (posterior horn facet, PHF). Anteriorly there is a second surface which may be approximated to a $50^{\circ}$ arc of a second circle with a larger radius $(32 \mathrm{~mm})$, the extension facet (EF, center: the EFC). The 


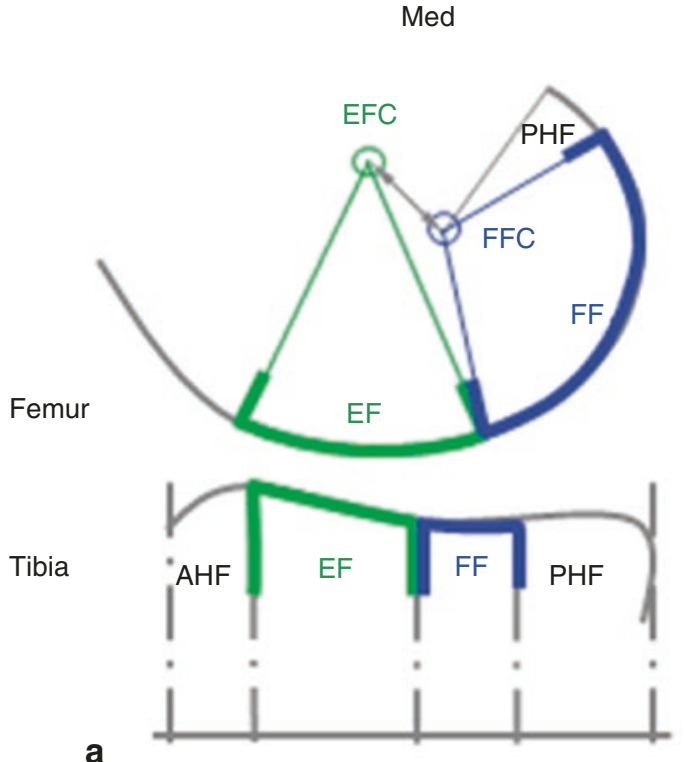

Fig. 14.1 Sagittal sections through the center of the medial (a) and lateral (b) compartments (see text) medial tibial surface, if sectioned centrally, can be seen to be posteriorly flat and horizontal over approximately $25 \mathrm{~mm}$ (the flexion facet, FF). The posterior $15 \mathrm{~mm}$ of this surface always contacts the posterior horn of the meniscus (posterior horn facet, PHF). Anteriorly the surface slopes $11^{\circ}$ upward and forward (the extension facet, EF) to contact the anterior circular surface of the femur in extension. Lateral femorotibial compartment: Laterally, the femur also has a posterior circular surface (FF, see Fig. 14.1b) subtending on average $114^{\circ}$ with a radius of $21 \mathrm{~mm}$. Anteriorly, the extension facet is much shorter compared to the medial condyle and therefore difficult to distinguish. The extreme posterior part of the femoral condyle (PHF) again contacts only the posterior horn of the meniscus, never the tibia. The extreme anterior end of the articular surface is relatively flat and contacts the anterior horn and the anterior extremity of the tibial articular surface in full extension (AHF). The central $24 \mathrm{~mm}$ of the lateral tibial surface is relatively flat (tibial articular facet, TAF). Anteriorly and posteriorly, the surface curves downward to receive the horns of the meniscus in extension and flexion (AHF,

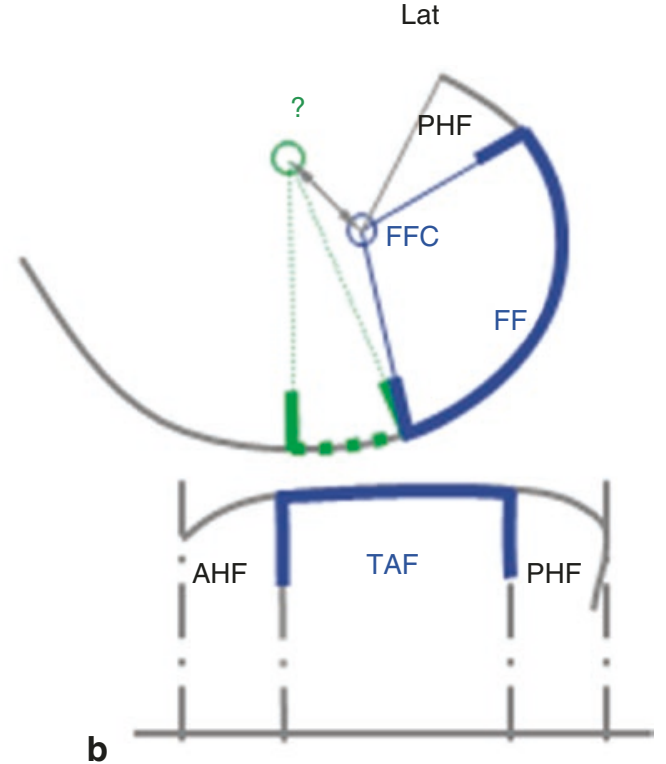

PHF), enhancing the impression of upward convexity, usually described as upwardly convex.

Collateral ligaments: Collaterals differ in the position of their attachment to the femur. The medial epicondyle with the medial collateral ligament (MCL) attachment coincides with the penetration point of the EFC. Medially, at first, contact occurs between the femoral and the tibial extension facets, the femur rotating on the tibia around the EFC axis and, therefore, around the MCL attachment (Fig. 14.2a). At about $30^{\circ}$ flexion, contact "rocks" onto the flexion facets, and the femur then rotates around the FFC axis (Fig. 14.2b). From then on, the MCL attachment rotates upward and backward around the FFC. The lateral collateral ligament (LCL) is attached to the femur at the lateral epicondyle. This coincides with the center of the femoral flexion facet, i.e., the entry point of the transcondylar axis. At full extension, the LCL is tight. As the knee flexes, there is backward motion of the lateral femoral condyle. The LCL becomes more vertical with flexion up to about $90^{\circ}$, the ligament being visibly slack. With flexion to $120^{\circ}$, the femoral condyle "drops" as it rolls over the 

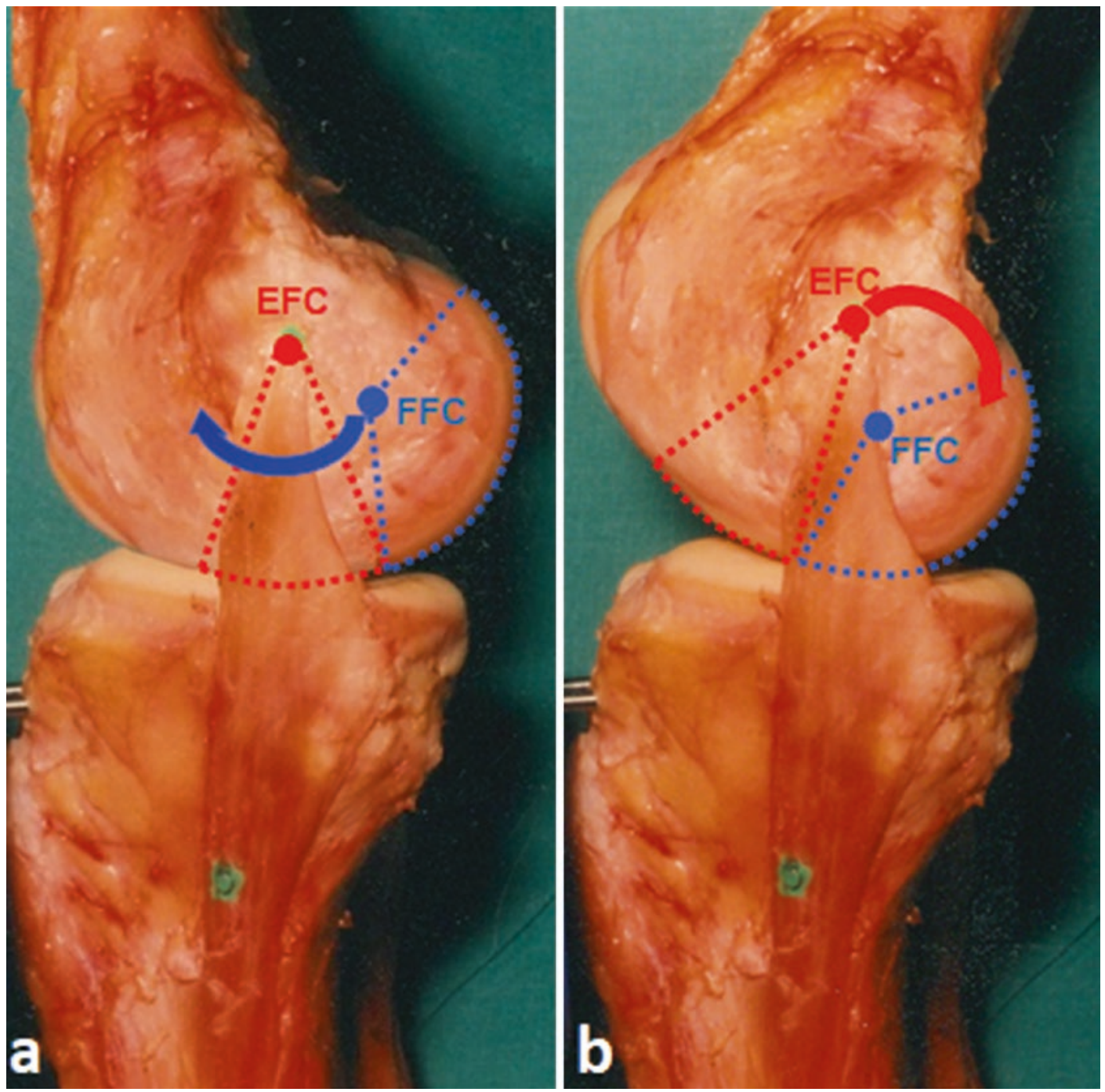

Fig. 14.2 Specimen viewed from the medial side in extension (a) and at $30^{\circ}$ flexion (b), showing the position of the MCL femoral attachment (see text)

posterior round surface of the tibia, further relaxing the LCL. As a result, collaterals differ in that the MCL (at least its anterior superficial portion [6]) remains tight during flexion, whereas the LCL relaxes with flexion.

The relative movement of the condyles: The movement of the knee has been considered in this review in the context of the lower limb as a whole. Considered in isolation, descriptions of knee movement in terms of rotations, translations, and axes may be given either as the position of the contact areas or as the movement of the condyles. This section deals with the latter. The movement of the knee can be divided into three functional arcs: terminal extension [1], arc of active flexion [2], and arc of passive flexion [3].

Terminal extension: This begins at the subject's limit of passive extension. This varies from about $5^{\circ}$ flexion to about $5^{\circ}$ hyperextension. The arc has peculiarities: the contact surfaces differ from those in the arc of active flexion; there is thought to be a near-obligatory association between longitudinal rotation and flexion. There is always a tendency toward internal femoral rotation. MR images show that rotation, when it occurs, is due to continued forward movement of the lateral femoral con- 
dyle, while the medial femoral condyle does not move anteroposteriorly. To achieve full extension, the medial femoral condyle must "rock" up onto the upward-sloping tibial condyle (Fig. 14.3a); the lateral femoral condyle rolls forward onto the flat tibial surface (Fig. 14.3b). Finally, as terminal rotation ends with both condyles immobilized anteroposteriorly, it obviously helps to stabilize the knee.

Arc of active flexion: In the arc of active flexion, the medial femoral condyle can be viewed as a sphere which rotates to produce a variable combination of flexion, longitudinal rotation, and minimal varus (if lift off occurs laterally). It hardly translates and thus is analogous to a somewhat constrained ball-in-socket joint. The lateral condyle rolls but also "slides" anteroposteriorly. This permits longitudinal rotation around an axis passing through the center of the medial sphere (the FFC) and flexion around an axis penetrating the two FFCs (because the femoral surfaces are circular and remain in contact with the tibia). The medial femoral condyle translates no more than $7.1 \mathrm{~mm}$ anteroposteriorly, weight bearing and non-weight bearing [7]. The lateral femoral condyle also rotates around its FFC, but in contrast to the medial side, it tends to translate posteriorly about $15 \mathrm{~mm}$ by a mixture of rolling and sliding $[7,8]$. As a consequence, between $10^{\circ}$ and $120^{\circ}$, the femur tends to rotate externally (tibia internally) about $30^{\circ}$ around a medial axis (Fig. 14.4). In the living weight-bearing knee during a squat, the general pattern of motion is again the same, although backward movement of the lateral femoral condyle may occur earlier [9]. At $90^{\circ}$, the tibia is free to rotate $20^{\circ}-30^{\circ}$ longitudinally without accompanying flexion.

Arc of passive flexion: This begins in a transition zone from $110^{\circ}$ to $120^{\circ}$ and continues to whatever may be the passive limit of the knee under study. The arc is entirely passive; the thigh muscles can flex the knee only to about $120^{\circ}$ against gravity. In the range of flexion from $120^{\circ}$ to $160^{\circ}$, the flexion
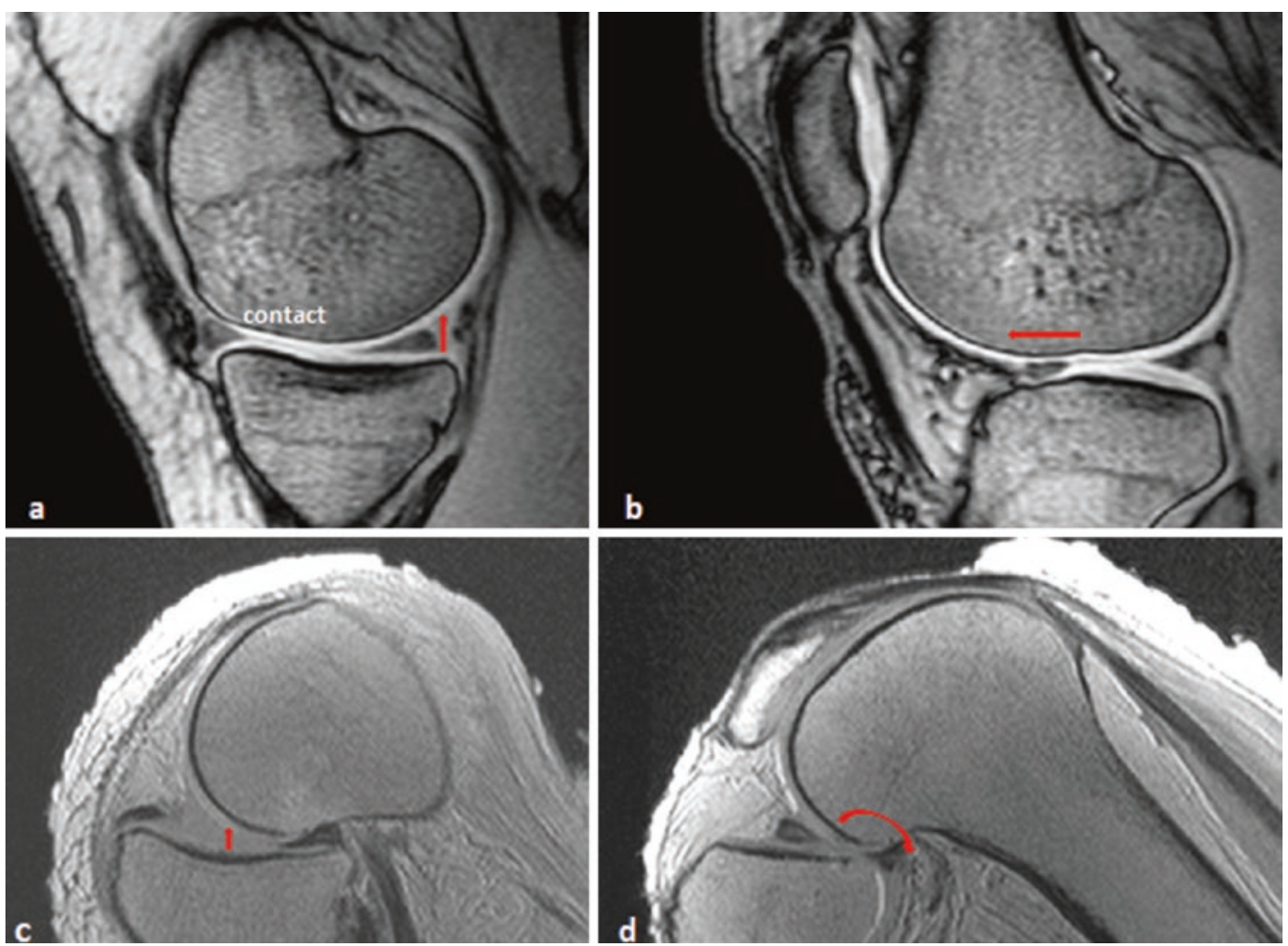

Fig. 14.3 Sagittal MRI through the medial (a) and the lateral compartments (b) in hyperextension (see text); sagittal MRI through the medial (c) and the lateral (d) compartments in $140^{\circ}$ flexion (see text) 


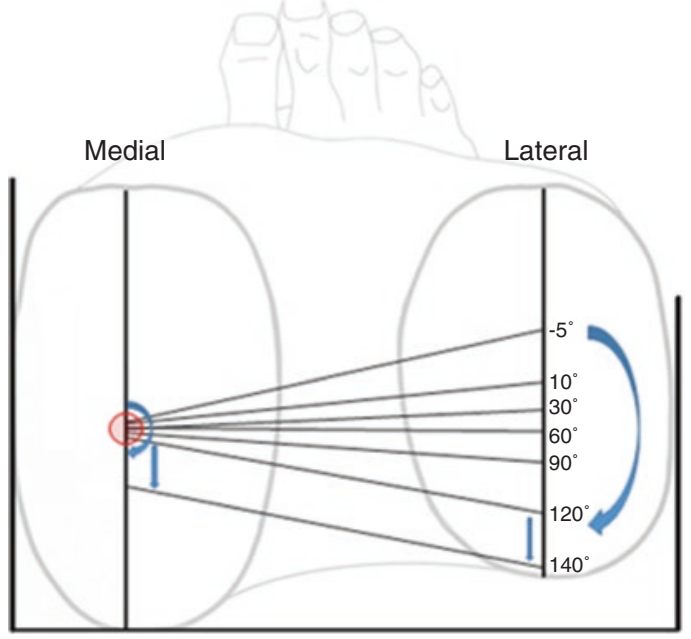

Fig. 14.4 Top of the tibia diagram showing the position of flexion axis from $-5^{\circ}$ to $140^{\circ}$ flexion (see text)

facet center of the medial femoral condyle moves back about $5 \mathrm{~mm}$ and rises up on to the posterior horn of the medial meniscus. At $160^{\circ}$, the posterior horn is compressed in a synovial recess between the femoral cortex and the tibia (Fig. 14.3c). This limits flexion. The lateral femoral condyle also rolls back, with the posterior horn of the lateral meniscus moving with the condyle. Both move down over the curved posterior border of the tibia (Fig. 14.3d). Neither the events between $120^{\circ}$ and $160^{\circ}$ nor the anatomy at $160^{\circ}$ could result from a continuation of the kinematics up to $120^{\circ}$. Therefore, hyperflexion is a separate arc of flexion. The anatomical and functional features of this arc suggest that it would be difficult to design an implant for total knee replacement that would enable physiological movement from $0^{\circ}$ to $160^{\circ}[10]$.

The Position of the Instant Axes from Full Extension to $160^{\circ}$ Flexion: The femoral condyles are composed of two circular arcs (the EF and the FF), these arcs forming the articular surfaces. The instant axes of flexion pass through their centers (the EFC and the FFC). The anteroposterior orientation of the axes is measured as their vertical position relative to the posterior tibial cortex. On the medial side, from full extension to $120^{\circ}$, motion is $96 \%$ sliding which makes it a reasonable approximation to locate the axes vertically at the geometrical center of the contacting femoral facet (i.e., the EFC or the FFC). Laterally, from full extension to $120^{\circ}$, there is about $40 \%$ rolling, suggesting that the axis lies about halfway between the center and the contact point. Anteroposteriorly the penetration point of the axis will be vertically below the geometrical center, that is, on a line perpendicular to the tibial contact surface. In the arc of passive flexion (from $120^{\circ}$ to $160^{\circ}$ ), as both femoral condyles roll back, the penetration points of the flexion axis appear to be at the posterior extremities of the medial and lateral femoral articular surfaces [10].

Longitudinal Rotation: Longitudinal rotation can be divided into that which accompanies flexion and that which occurs independently of flexion. We refer to rotation relative to the tibia of the femoral condyles, not to the flexion axes. The axis of longitudinal rotation is parallel to the long axis of the tibia. Zero might be defined as the rotational position of the femoral condyles at $0^{\circ}$ flexion. Alternatively, zero might be defined as the rotational position of the femoral condyles relative to the frontal plane. From extension to $120^{\circ}$ flexion, the medial femoral condyle does not move anteroposteriorly, while the lateral one moves posteriorly $18 \mathrm{~mm}$. Figure 14.4 shows a diagram of tibial condyles with lines representing the connection of the medial and the lateral FFCs from $-5^{\circ}$ to $140^{\circ}$ flexion. If the tibia is considered as fixed, the femur tends to rotate externally $20^{\circ}$. During the arc of the terminal extension, the femur rotates externally about $7^{\circ}$. There is thought to be a near-obligatory association between longitudinal rotation and flexion. In the arc of active flexion, the tibia (femur) is free to rotate $20-30^{\circ}$ longitudinally without accompanying flexion. Thus the tibial IR which usually accompanies flexion is not obligatory. During the passive arc of flexion, as the medial femoral condyle moves back about $3 \mathrm{~mm}$ more than the lateral one, a little femoral internal rotation occurs from $120^{\circ}$ to $160^{\circ}$ of flexion [10].

Varus/Valgus Rotation: In full extension, both collateral ligaments are tight, and varus/valgus rotation is therefore hardly possible. As the knee flexes, the lateral collateral ligament becomes loose and enables not only longitudinal rotation around the medial axis but also varus rotation. Figure 14.5 shows the frontal section of the knee at $90^{\circ}$ flexion with varus force applied to the tibia. The lateral joint space opens by a mean of $6.7^{\circ}$ 


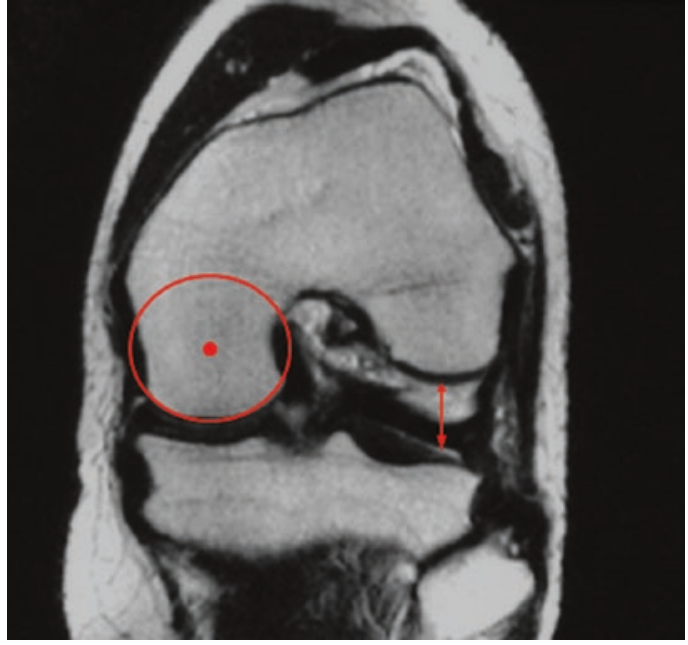

Fig. 14.5 Frontal MRI of a volunteer's knee at $90^{\circ} \mathrm{flex}-$ ion, with varus stress applied. For reasons explained in the text, the LCL is slack, and therefore the femur can be separated from the tibia laterally (so-called lift off). The medial joint gap under valgus stress opens only by a mean of $2.1 \mathrm{~mm}$ [11]. There is therefore a clear asymmetry between the lateral and medial flexion gaps. This asymmetry can be explained both by the laxity of the LCL and by the shapes of the articulating bones. In the coronal plane, the posterior portion of the medial femoral condyle is also spherical; therefore, varus rotation occurs around the axis passing through the center of this sphere. In full flexion, valgusvarus movement was measured in volunteers whose knees were fully flexed and stressed manually into tibial valgus and varus [10]. No movement was detected on valgus stress, but the lateral compartment opened up to $10 \mathrm{~mm}$ under varus stress. These findings would be expected from the observed tensions in the collateral ligaments.

Finally, in the arc of active flexion, we can define three axes of motion (Fig. 14.6) which

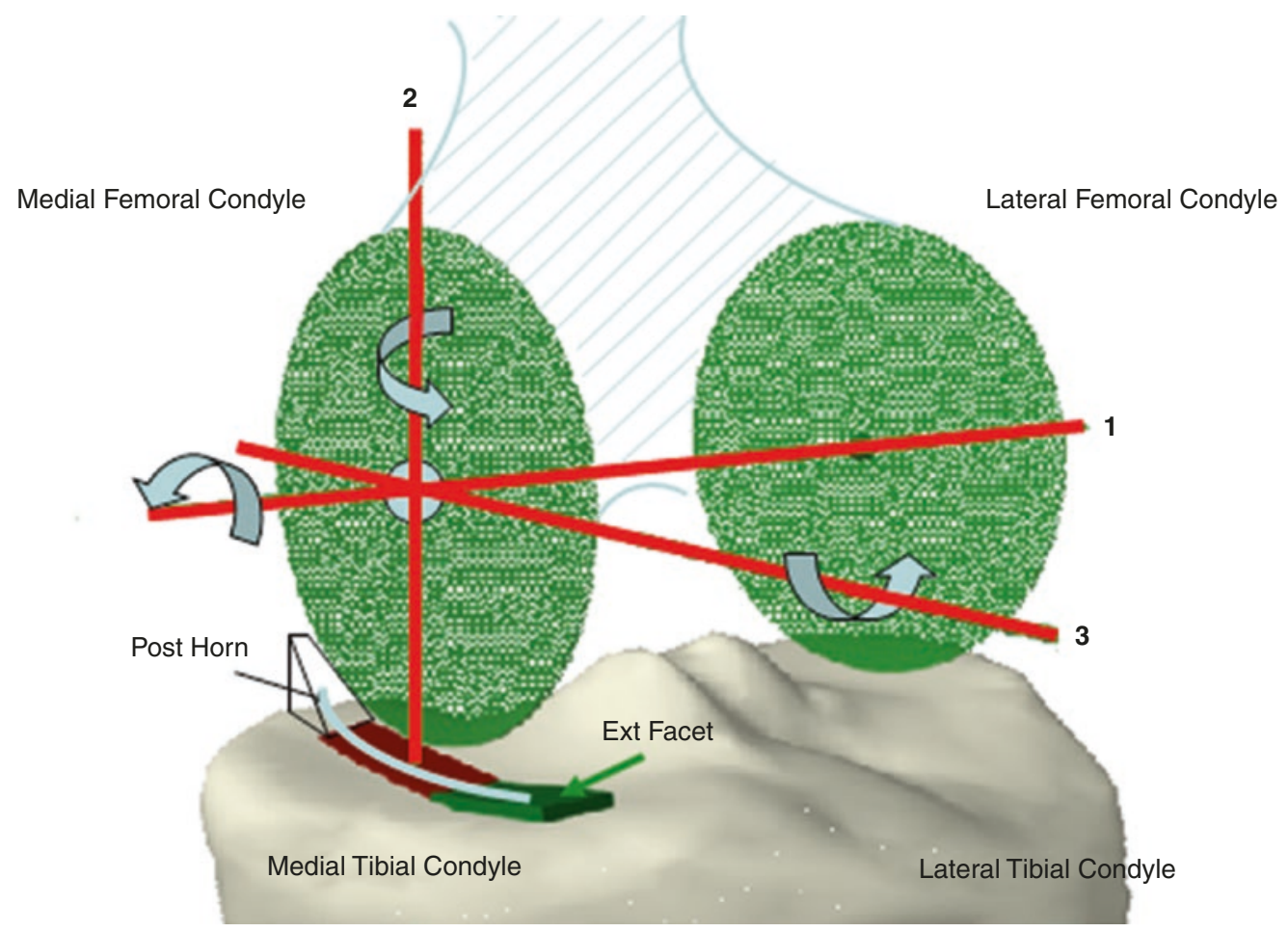

Fig. 14.6 Model of the knee showing the orientation of the three axes of motion in the active arc of flexion: 1 , flexion axis; 2, longitudinal rotation axis; and 3, varus/ valgus axis. They intersect roughly in the center of the posterior portion of the medial femoral condyle 
are perpendicular to each other. They intersect around the center of the posterior portion of the medial femoral condyle (i.e., FFC).

\subsection{How Inter-Individually Variable Is Knee Anatomy?}

There is high individual variability in the tibiofemoral joint with respect to the anatomy, tissue properties, joint kinetics, and kinematics. It is influenced by both intra-articular and extraarticular parameters.

Increased attention has been paid to the shape, in particular, with respect to race and gender differences, of the distal femur. Data from the literature suggest that the female knee is narrower than the male knee, regardless of its size $[12,13]$. It has been reported that standard TKA (total knee arthroplasty) used for a narrow female knee results in ML overhang of the femoral component [14]. Bellemans et al. [15] suggested that there are factors other than gender which also have an influence on the shape of the knee and that there is variability within each gender which could be explained by morphotypic variation. The morphotype characterization was based on the pelvis width/total leg length ratio. Patients with short and wide morphotype (endomorph) had, irrespective of gender, wider knees, whereas patients with long and narrow morphotype (ectomorph) had narrower knees. Morphotype significantly predicted the femoral aspect ratio but only weakly predicted the tibial aspect ratio. Lancaster and Nunley $[16,17]$ report a wide variation in the angle between extension and flexion facets of the medial tibial condyle (EFA) in normal knees that is unrelated to age. There is an association between an increased EFA (i.e., a steeper extension facet) and MRI evidence of anteromedial osteoarthritis. Although a causal link is not proven, Lancaster speculates that a steeper angle increases the duration of loading on the $\mathrm{EF}$ in stance and tibiofemoral interface shear. Eckhoff et al. [18] suggest there is a variation in the so-called "version" of the knee, defined as the static rotation of the tibia with respect to the femur in full knee extension. This angle, repre- senting external rotation of the tibia relative to the femur, was increased significantly in patients with anterior knee pain. In conclusion, there is high individual variability in tibiofemoral joint conformity, and a wide range of TKA component sizes and shapes would be required to accurately replicate native joint conformity in most people.

\subsection{When to Re-create and When Not to Recreate the Constitutional Knee Anatomy?}

It follows that, firstly, when describing movement of the knee, care must be taken to define whether it is the contact points or the condyles that are under investigation since the two move differently and the movement of the bones cannot be deduced solely from the contact areas; secondly, that while it may be possible to design a total knee replacement implant which could replicate either the movement of the condyles or the contact areas, to replicate both, i.e., to produce normality, is probably impossible.

Key to a successful outcome of TKA is to achieve correct alignment, proper balance, and deformity correction.

Mechanical alignment in TKA introduced by Insall et al. [19] was used to achieve an even load distribution on the new joint line. However, part of the patients is still disappointed with the outcome. Bellemans [20] reports there is increasing evidence that for a number of patients, neutral alignment is not normal. For patients with so-called constitutional varus, restoring neutral alignment may not be the best option since it is abnormal to them. The development of a kinematic alignment aims to provide a properly balanced TKA during the whole arc of motion. The concept of kinematic alignment is to restore normal knee function by aligning the distal and posterior femoral joint line of the femoral component according the functional femoral transverse axes and joint line of the tibial component to those of the normal or pre arthritic condition.

This is possible in primary osteoarthritis without severe bone defects and ligament laxity. 
Resurfacing the knee joint with kinematic alignment by preserving normal ligament laxities may be an attractive option in these cases.

However, certain circumstances such as posttraumatic arthritis or rheumatoid arthritis are often associated with severe deformities, significant bone defects, contractures, and instability. Such deformities are extremely difficult to balance with soft tissue release only and require additionally constrained prostheses even in primary TKA. To correct varus deformity, various techniques including step releases and multiple needle puncturing of the medial soft tissue structures during TKA are in common use. Correctly tensioned MCL represents the fundamental condition of restoring normal knee kinematics. Using anatomic designs after adequate medial release is therefore a method of choice because the primary stabilizer of the medial compartment is preserved.

Correction of fixed valgus deformity during TKA presents a challenging task. Multiple techniques to restore limb alignment and correct instability have been described, including various techniques of lateral soft tissue release, lateral femoral sliding, epicondylar osteotomy, reconstruction of the medial collateral ligament, and finally the use of a constrained condylar implant.

Little data exists to describe the kinematics of severe valgus knee. Baier [21] describes a paradoxical longitudinal rotation during flexion, suggesting that valgus knee rotates around the lateral axis. On the assumption that collateral ligaments behave conversely during flexion (i.e., MCL is loose and LCL remains tight), it would be extremely difficult, if not impossible, to restore natural knee kinematics using medially rotating knee designs. In such a situation, constrained condylar knee designs are the ultimate choice.

\subsection{Can the Natural Knee Kinematics Be Reproduced in TKA?}

Total knee arthroplasty has now been performed for more than five decades. The procedure has been successful in relieving patients', pain but both orthopedic surgeons and patients continue to seek a better functional outcome. However, a "forgotten" knee after TKA is unusual, whereas a "forgotten" hip after total hip replacement is commonplace.

It has been shown in multiple in vivo analyses that the kinematic patterns after mechanically aligned TKA differ considerably from those of the normal knee [22]. Pritchett [23] analyzed patients' preferences in mechanically aligned knee replacement. In a group of 688 of bilateral knee arthroplasty recipients (after excluding poor results), most of the patients thought one knee was worse than the other. They attributed this to the feeling of the inferior knee being less normal, weaker, on stairs, or less stable. It is interesting that the preferred knees all had a-p stable design: all of the patients preferred either the retention of both cruciates with the use of an ACL-PCL prosthesis or a substitution with medially pivoting designs.

Conventional designs of knee replacements, i.e., the posterior cruciate ligament (PCL) retaining and PCL substituting knees, failed to reproduce normal knee kinematics. Anterior cruciate ligament deficiency after TKA causes femoral forward motion during knee flexion, the so-called paradoxical motion. However, too much joint laxity is associated with persistent pain and poor long-term outcomes as a result of instability, causing premature polyethylene wear.

To improve anteroposterior stability, bicruciate-retaining total knee arthroplasty was introduced. However, it did not gain widespread popularity over recent decades because of unpredictable tensioning of the retained cruciate ligaments. Too high or low tension in the ACL can cause knee stiffness or instability after bicruciateretaining TKA [24].

To reduce abnormal strains at the boneimplant interface during motion, mobile-bearing knee replacements based on a mobile polyethylene insert that articulates with a metallic femoral component and a metallic tibial tray were introduced.

However, relatively high rate of mechanical complications (including loosening of the femo- 
ral component, tibiofemoral dislocation in high flexion, and insert breakage) was described [25].

The stability and the kinematics of the knee depend on the musculature, the surrounding ligaments, the implants' orientation, and the geometry of the articular surfaces. Since the TKA procedure is nowadays performed on younger, more active patients, proper anteroposterior stability, and natural axial rotation patterns are essential for good patellar tracking and improved knee flexion.

TKA design should therefore provide anteroposterior stability and simultaneously allow longitudinal rotation, i.e., reproduce the pattern of movement of the natural knee. The concept, based on ball-in-socket geometry medially and with less constrained lateral surface, enables longitudinal rotation around the medial axis. This movement is possible because the LCL is loose in flexion. The combination of fully congruent medial compartment and flat lateral tibial surface, together with tight MCL and loose LCL, enables flexion accompanied with femoral external rotation around the stable medial condyle. The increased contact area reduces contact stresses and subsequent linear polyethylene wear.

Fully congruent medial designs reproduce neither the normal knee anatomy nor the motion toward full extension. Nevertheless, reproducing the anterior "rocking" of the medial femoral condyle onto the tibial extension facet toward full extension in TKA would increase the loading of the anterior lip of the tibial insert, causing excessive polyethylene wear. In deep flexion, the medially conforming articulation is beneficial in controlling the femoral AP position.

With the improvement of imaging and image processing technologies, the patient-specific cutting guides and patient-specific implants developed with the aim to create articular surfaces which closely mimic natural anatomy and kinematics of the knee.

In conclusion, providing stable and consistent knee kinematics in total knee replacement is an essential requirement of good long-term clinical results.

\section{References}

1. Zuppinger H. Die aktive flexion im unbelasteten Kniegelenk. Bergmann: Züricher Habil. Schr. Wiesbaden; 1904. p. 703-63.

2. Brantigan OC, Voshell AF. The mechanics of the ligaments and menisci of the knee joint. J Bone Joint Surg. 1941;23:44.

3. Iwaki H, Pinskerova V, Freeman MAR. Tibio-femoral movement 1: the shapes and relative movements of the femur and tibia in the unloaded cadaver knee. $\mathrm{J}$ Bone Joint Surg. 2000;82B(8):1189-95.

4. Martelli S, Pinskerova V. The shapes of the tibial femoral articular surfaces in relation to tibiofemoral movement. J Bone J Surg. 2002;84B:607-13.

5. McPherson A, Karrholm J, Pinskerova V, Sosna A, Martelli S. Imaging knee motion using MRI, RSA/CT and 3D digitization. J Biomech. 2005;38(2):263-8.

6. Gardiner JC, Weiss JA, Rosenberg TD. Strain in the human medial collateral ligament during valgus loading of the knee. Clin Orthop Rel Res. 2001;391:266-74

7. Johal P, Williams A, Wragg P, Gedroyc W, Hunt M. Tibio-femoral movement in the living knee. An in-vivo study of weight bearing and non-weight bearing knee kinematics using 'interventional' MRI. J Biomech. 2005;38(2):269-76.

8. Kurosawa H, Walker PS, Abe S, Garg A, Hunter T. Geometry and motion of the knee for implant and orthotic design. J Biomech. 1985;18(7):487-99.

9. Hill PF, Vedi V, Iwaki H, Pinskerova V, Freeman MAR, Williams A. Tibio-femoral movement 2: the loaded and unloaded living knee studied by MRI. J Bone Joint Surg. 2000;82B(8):1196-8.

10. Pinskerova V, Samuelson KM, Stammers J, Maruthainar K, Sosna A, Freeman MAR. The knee in full flexion an anatomical study. J Bone Joint Surg. 2009;91B(6):830-4.

11. Tokuhara Y, Kadoya Y, Nakagawa S, Kobayashi A, Takaoka K. The flexion gap in normal knees: a MRI study. J Bone Joint Surg. 2004;86B:1133-6.

12. Dargel J, Joern WPM, Feiser J, Ivo R, Koebke J. Human knee joint anatomy revisited: morphometry in the light of sex-specific Total knee arthroplasty. J Arthroplast. 2011;26(3):346-53.

13. Guy SP, Farndon MA, Sidhom S, Al-Lami M, Bennett C, London NJ. Gender differences in distal femoral morphology and the role of gender specific implants in total knee replacement: a prospective clinical study. Knee. 2012;19(1):28-31.

14. Koninckx A, Deltour A, Thienpont E. Femoral sizing in total knee arthroplasty is rotation dependent. Knee Surg Sports Traumatol Arthrosc. 2014;22(12):2941-6.

15. Bellemans J, Carpentier K, Vandenneucker $H$, Vanlauwe J, Victor J. The John Insall Award. Both morphotype and gender influence the shape of the knee in patients undergoing TKA. Clin Orthop Relat Res. 2010;468:29-36. 
16. Lancaster BJA, Cottam HL, Pinskerova V, Eldridge JDJ, Freeman MAR. Variation in the of the tibial plateau. A possible factor in the development of anteromedial osteoarthritis of the knee. J Bone Joint Surg. 2008;90B(3):330-3.

17. Nunley RM, Nam D, Johnson SR, Barnes CL. Extreme variability in posterior slope of the proximal tibia: measurements on 2395 CT scans of patients undergoing UKA. J Arthroplast. 2014;29:1677-80.

18. Eckhoff DG, Brown AW, Licoyne RF, Stamm ER. Knee version associated with anterior knee pain. Clin Orthop Relat Res. 1997;339:152-5.

19. Insall JN, Binazzi R, Soudry M, et al. Total knee arthroplasty. Clin Orthop Relat Res. 1985;192:13-2.

20. Bellemans J. Neutral mechanical alignment: a requirement for successful TKA: opposes. Orthopedics. 2011;34:e507-9.
21. Baier C, Benditz A, Koeck F, Keshmiri A, Grifka J, Maderbacher G. Different kinematics of knees with varus and valgus deformities. J Knee Surg. 2018;31(3):264-9.

22. Blakeney W, Clément J, Desmeules F, Hagemeister N, Rivière $\mathrm{C}$, Vendittoli PA. Kinematic alignment in total knee arthroplasty better reproduces normal gait than mechanical alignment. Knee Surg Sports Traumatol Arthrosc. 2019;27(5):1410-7.

23. Pritchett JW. Patient preferences in knee prostheses. J Bone Joint Surg. 2004;86B(7):979-82.

24. Okada Y, Teramoto A, Takagi T, et al. ACL function in bicruciate-retaining total knee arthroplasty. J Bone Joint Surg Am. 2018;100:e114(1-7) d.

25. Chang CW, Lai KA, Yang CY, et al. Early mechanical complications of a multidirectional mobilebearing total knee replacement. J Bone Joint Surg. 2011;93-B(4):479-83.

Open Access This chapter is licensed under the terms of the Creative Commons Attribution 4.0 International License (http://creativecommons.org/licenses/by/4.0/), which permits use, sharing, adaptation, distribution and reproduction in any medium or format, as long as you give appropriate credit to the original author(s) and the source, provide a link to the Creative Commons license and indicate if changes were made.

The images or other third party material in this chapter are included in the chapter's Creative Commons license, unless indicated otherwise in a credit line to the material. If material is not included in the chapter's Creative Commons license and your intended use is not permitted by statutory regulation or exceeds the permitted use, you will need to obtain permission directly from the copyright holder. 